\title{
ESTUDIOS SOCIALES SOBRE EL MAESTRO COLOMBIANO
}

\author{
Rodrigo Parra Sandoval*
}

Muy pocos temas dentro del sector educativo generan un consenso tan amplio sobre su importancia, sobre la necesidad y urgencia de realizar estudios en profundidad, como el de los maestros. Esta situación tiene, por supuesto su génesis en razones de tipo social y político. La actividad de los maestros afecta directamente a una buena proporción de la sociedad, la calidad de la enseñanza, sus costos, sus carencias son temas de frecuente discusión, los maestros se han organizado sindicalmente y ejercen presiones. Sin embargo, es paradójica la poca importancia que se le ha dado en Colombia al estudio del maestro en el área de las Ciencias Sociales. Por eso parece necesario detenerse un momento a mirar qué se sabe sobre ellos como actores sociales y, sobre todo, a pensar en lo que es necesario saber para obtener una comprensión básica de su problemática profesional y de sus interacciones con la sociedad en que trabajan.

La revisión de literatura sobre maestros que se presenta aquí tal vez no sea exhaustiva pero permite delinear unas tendencias y plantear unas líneas de trabajo para la investigación social del tema.

Las orientaciones teóricas, los énfasis disciplinarios y las metodologías usadas muestran una amplia dispersión, tal vez como efecto o como síntoma de la falta de continuidad de los estudios. No ha existido un estudio general que sirva de guía para organizar los trabajos subsiguientes con arreglo a sus planteamientos, ni un esfuerzo de priorizar las necesidades de investigación en este campo con propósitos ya sea académicos o de política educativa los estudios de mayor relieve no han tenido un seguimiento posterior que profundice, critique o amplíe sus resultados.

Desde el ángulo de los enfoques teóricos más frecuentemente utilizados sobresalen las siguientes tendencias:

1. Estudios con finalidades puramente descriptivas que narran situaciones concretas en lugares específicos. Aquí pueden catalogarse los estudios de comunidad y los trabajos sobre escuelas o maestros que no llevan su análisis más allá de caracterizar un objeto de estudio y que se enmarcan dentro de problemas más generales que sobrepasan el área puramente educativa.

2. Estudios sobre la profesión del maestro que se inscriben principalmente dentro de la Sociología de las profesiones.

3. Estudios con propósito explicativo que intentan llevar a cabo la conexión entre los fenómenos sociales más generales y aspectos concretos de la vida y quehacer profesional de los maestros.

4. Estudios que relacionan el proceso de desarrollo desigual con la educación y los maestros. Este parece ser el enfoque favorecido más frecuentemente, tal vez debido a

\footnotetext{
* Rodrigo Parra. Sociólogo de la Universidad Nacional, master en sociología de La Universidad de Wisconsin; Ph. D. en sociología, Universidad de Wisconsin, investigador CEDE Universidad de los Andes.
} 
la preponderante importancia de las diferencias regionales y rural-urbanas en Colombia.

5. Estudios que emplean planteamientos propios del análisis empirista, ahistóricos y abstractos y que se llevan a cabo por medio de diferentes técnicas de análisis multivariado.

Paradójicamente, el gran ausente teórico en el análisis del maestro (con pocas y honrosas excepciones), es el análisis social desde el punto de vista de las ideas pedagógicas, de sus formas de aplicación y de sus consecuencias en la enseñanza y en la formación de imágenes, actitudes y conductas en el alumno.

Las metodologías empleadas varían desde estudios etnográficos con énfasis descriptivo pasando por análisis de datos secundarios, encuestas originales sobre poblaciones restringidas, sobre grupos representativos de modelos teóricos o representativos de unidades poblacionales urbanas, hasta análisis con técnicas cuantitativas de mayor sofisticación. No existe un censo de maestros que permita sacar conclusiones con mayor grado de cobertura poblacional o extraer muestras con mayor rigor para realizar estudios más específicos y profundos. Las muestras empleadas en los estudios sobre maestros no permiten, en la mayoría de los casos, generalizar los hallazgos a poblaciones más amplias aunque pueden ser de mucha utilidad para sugerir hipótesis y caminos de investigación.

En general los trabajos publicados han sido realizados por centros privados de investigación, centros universitarios, Ministerio de Educación Nacional y entidades internacionales. Sus propósitos responden fundamentalmente a dos avenidas de intereses: la planeación educativa y la investigación básica, o una mezcla de ambas.

Con estas anotaciones generales se puede entrar a la presentación de algunos de los temas tratados en los trabajos.

\section{Los maestros y el desarrollo del país}

En realidad no son muy numerosos los trabajos encontrados sobre el maestro y su relación con la sociedad colombiana. La mayor parte de la información contenida en los estudios sociales se encuentra en trabajos sobre temas más generales en los que se incluye al maestro como un elemento del desarrollo del país, cuyas características históricas de múltiples deficiencias tanto cuantitativas como cualitativas lo convierten en un obstáculo para dicho proceso ${ }^{18}$. Otro tipo de estudio enfoca al maestro como agente de cambio y trata de formular los caminos por medio de los cuales este proceso se lleva a cabo $^{19}$.

En términos más generales, pero siguiendo la misma directriz, se enfoca la relación entre la acción del maestro y su sociedad desde dos avenidas opuestas: desde los factores sociales que hacen posible u obstaculizan su función de agente de cambio o desde las acciones que desempeña el maestro ${ }^{20}$ y su efecto en cambiar el medio social y

\footnotetext{
${ }^{18}$ Por ejemplo el trabajo de la Misión Economía y Humanismo, Estudio sobre las condiciones del desarrollo de Colombia, Bogotá, Presidencia de la República, 1958. Banco Mundial, Desarrollo económico de Colombia, Bogotá, Banco Popular, 1970.

${ }^{19}$ Dieter Pass, El maestro rural, Bogotá, Fundación Friedrich Naumann, 1979.

${ }^{20}$ Wilson Velandia, et al., La educación no formal en Colombia, Bogotá, CEDEN, 1975.

Rodrigo Parra, Bases sociales para la formación de los maestros colombianos, CIUP-UPN y CEPAL-UNESCO-PNUD, proyecto 'Desarrollo y Educación en América Latina y el Caribe' '1979. La profesión del maestro y el desarrollo nacional, Ministerio de Educación, CIUP-UPN, UNESCO-CEPAL-PNUD, proyecto "Desarrollo y Educación en América Latina y el Caribe", 1979, mimeo. 
catalizar las fuerzas que llevan hacia su desarrollo ${ }^{21}$. Estos planteamientos se hallan generalmente subyacentes en los trabajos y muy pocas veces se expresan de manera explícita en los análisis.

Por supuesto ambas formas de analizar el problema muestran una parte de la realidad. Queda, sin embargo, por plantearse en forma totalizante las relaciones que se dan entre ambos procesos, la manera en que se entrelazan las dos caras de la moneda.

De manera más específica el tema de las relaciones entre la actividad profesional de los maestros y el proceso de desarrollo del país ha sido enfocado fundamentalmente desde teorías que tienden a privilegiar el examen de la desigualdad del crecimiento dentro del país. Esta línea de estudio parece especialmente aplicable al caso colombiano debido a la gran diversidad cultural, económica y de situaciones sociales de sus regiones. Parece entonces de interés revisar algunas de las líneas de investigación que se han utilizado.

1. Los trabajos de Ferro ${ }^{22}$ y Pass analizan o muestran, aunque de manera no explicita los problemas que encuentran los maestros como resultante de los cambios de una región a otra. de una escuela a otra, de sus contactos con comunidades, de la enseñanza de la geografía y la historia de las localidades y de su adaptación de la docencia a necesidades especificas. Estos estudios se centran en la visión que expresan los maestros de los problemas que causa el cambio de escuela. En este grupo se pueden clasificar también los estudios de comunidades tanto rurales como indígenas que incluyen el tratamiento de algunos aspectos de la relación entre maestros y la comunidad.

2. Un segundo grupo de trabajos es el que se orienta hacia el análisis de recursos humanos en regiones específicas, a establecer subdivisiones intrarregionales con propósitos de servir de base a la planificación educativa y cuyo énfasis, en lo que se refiere a los maestros, está puesto en el análisis de las características de los docentes, tanto reales como necesarias con el objetivo de adoptar medidas de política que los haga más adecuados. Aquí cabría mencionar los trabajos de OFISEL y, de alguna manera, el de Cataño ${ }^{23}$.

3. El estudio de W. Velandia ${ }^{24}$, en lo que respecta a la relación entre desarrollo y docentes, se centra en observar cómo las variaciones del desarrollo en diferentes regiones, los procesos de urbanización y de crecimiento económico de los diferentes espacios estudiados, inciden en la naturaleza de los programas de educación no formal, en las características de los maestros y en sus técnicas pedagógicas. De la misma manera, aunque con explicitación de las bases teóricas empleadas, el trabajo de Parra sobre la profesión del maestro dirige su análisis hacia la situación planteada por la existencia de un currículum único de educación primaria y sus consecuencias en seis diferentes contextos sociales con grados diferentes de desarrollo. Se observan desde este ángulo las características educativas, migratorias, de capacitación, de imagen profesional, en los contextos sociales y se extraen líneas generales de política para la capacitación.

\footnotetext{
${ }^{21}$ En general los trabajos realizados dentro del rubro estudios de comunidades que se citan en este ensayo.

${ }_{22}^{22}$ Myriarn Stella Ferro, Mi vida: historia de la vida de una maestra rural colombiana, Bogotá, América Latina. 1978.

${ }^{23}$ Gonzalo Cataño, La educación rural en Boyacá: inventario de problemas, Tunja, Universidad Pedagógica y Tecnológica de Colombia, 1971. OFISEL, Regionalización y educación primaria en áreas rurales de Boyacá, Universidad Pedagógica y Tecnológica de Colombia, Tunja. 1976.

${ }^{24}$ Wilson Velandia. op. cit. 
4. Una cuarta línea de análisis enfatiza los condicionamientos que procesos sociales concretos relacionados con el desarrollo del país ejercen sobre fenómenos educativos que se vinculan directamente a la actividad docente. Se presentan interpretaciones del proceso de integración nacional tanto a nivel político como económico, conceptos sobre la génesis del desarrollo desigual y sus evoluciones, de la transmisión del concepto de nacionalidad y de factores sociales que impiden el desarrollo del magisterio como profesión ${ }^{25}$.

Estos estudios y la información empírica que contienen, aunque dan ya algunas luces sobre las relaciones entre maestro y desarrollo de la sociedad, apenas empiezan a colocar las primeras piezas del rompecabezas. Algunos de sus hallazgos concretos de mayor relevancia se presentan en los apartes siguientes.

\section{Desarrollo económico y expansión de las escuelas normales}

En 1958 el informe de la misión Economía y Humanismo planteaba como el problema más agudo del sistema educativo colombiano el alto déficit de maestros para las necesidades de la población ${ }^{26}$. En las dos décadas siguientes este problema iba a experimentar un vuelco fundamental. Parece entonces importante mirar las condiciones económicas y sociales que llevaron a este cambio y los efectos que dicho cambio produjo en la naturaleza de las normales. Se presentan en seguida las ideas centrales de un trabajo que, ha tratado este punto ${ }^{27}$.

En este trabajo se presenta un análisis somero de las directrices que ha tomado el proceso de desarrollo del país a partir de la cuarta década del presente siglo. Se trata fundamentalmente de lo que se ha denominado el proceso de integración económica del espacio nacional, iniciado a partir de la inserción del país a la economía internacional con el café como producto exportable e implementado más directamente por la industrialización del sistema productivo y sus consecuencias en la urbanización y en la configuración de un sistema jerarquizado y concentrador del desarrollo. Esa tendencia del desarrollo se vincula con lo sucedido en las instituciones encargadas de la formación de los docentes colombianos: las escuelas normales.

Durante el período comprendido entre 1939 y 1975 la evolución de los estudios normalistas muestra dos claras etapas:

1. Una etapa de rápido crecimiento y expansión no solamente numérica sino espacial de las normales, la matrícula y los profesores de normal.

2. Una etapa de disminución del crecimiento en que las tasas de crecimiento de las normales y los profesores normalistas muestran una tendencia decreciente al tiempo que también disminuye el ritmo de crecimiento de la matrícula.

Ambas etapas tienen consecuencias de mucha importancia para la definición de lo que es hoy la educación normalista y por esto se analizan en seguida algunos de los fenómenos que las caracterizan.

\footnotetext{
${ }^{25}$ E. A. Havens y W. L. Flinn, "Structural Blocks to Higher Educational Attainment “, en E. A. Havens y W. L. Flinn, Internal Colonialism and Structural Change in Colombia, New York, Praeger, 1970.

${ }^{26}$ Misión Economía y Humanismo, op. cit.

${ }^{27}$ Rodrigo Parra, Bases sociales, op. cit.

Digitalizado por RED ACADEMICA
} 
En los veinticinco años que van entre 1939 y 1965 el número de escuelas normales creció 985 veces, el número de maestros que trabajan en escuelas normales se multiplicó por 809 y el número de alumnos matriculados en escuelas normales se hizo 1.545 veces mayor. Un crecimiento de tal magnitud tuvo que venir acompañado de fenómenos sociales y educativos de importancia correlativa.

Como resultado de estos fenómenos la educación normalista experimentó cambios fundamentales en su naturaleza en por lo menos dos direcciones: Sufrió un fuerte proceso de privatización impulsada por la fuerte demanda y por la insuficiente respuesta del sector público y un notable acentuamiento de su feminización.

El crecimiento de las normales respondió básicamente a las demandas condicionadas, en un lado por la expansión de la matrícula por el surgimiento de clases medias urbanas, y en el otro por determinantes ideológicos que daban valoraciones positivas a la vinculación de la mujer al mercado de trabajo en áreas similares a su papel tradicional, una especie de continuación fuera de la institución familiar, de su papel de madre y educadora.

Entre 1965 y 1974 la matrícula en escuelas normales reduce drásticamente la velocidad de sus tasas de crecimiento. Este fenómeno sugiere, parcialmente, la saturación del mercado de trabajo de los maestros, y vino acompañado de dos hechos de la mayor importancia. Uno de ellos es la rápida aceleración de las tasas de crecimiento de la matrícula secundaria, especialmente del bachillerato, y el otro es la expansión de la matrícula en universidades pedagógicas.

Estos dos hechos, que pueden haber gravitado positivamente en los niveles de calidad del magisterio, marcan al tiempo una intensificación del carácter urbano de la formación de los maestros, refuerzan la visión urbano-industrial de la educación y trasladan cada vez con más fuerza a la ciudad las posibilidades de movilidad social por medio de la educación.

La disminución de la importancia relativa de la matrícula en escuelas normales tiene implicaciones tanto a nivel del desarrollo regional como a nivel del papel que le cabe jugar a los sexos en el proceso. En el plano espacial este fenómeno implicó una pérdida de concentración para los cinco departamentos más industrializados que ahora tienen solamente el $50 \%$ de la matrícula total, el $46 \%$ de la oficial y el $58 \%$ de la privada. La disminución se debió centralmente a la declinación de matrícula en el sector privado que pasó de representar el $74 \%$ al $58 \%$, al tiempo que la proporción de matrícula oficial permanecía prácticamente inalterada.

Los departamentos más industrializados tienden a tener no solamente las normales mejor calificadas sino también una buena proporción de las normales de inferior calidad. La primera parte del fenómeno descrito es parte de la concentración educativa que conlleva el proceso de desarrollo desigual, y la segunda se debe a la naturaleza de la expansión de la enseñanza normalista discutida anteriormente.

A nivel urbano este hecho está marcando una estratificación de las normales, lo que posiblemente tenga incidencia no solamente en la calidad de los egresados y de la enseñanza posterior que ellos lleven a cabo, sino también posibilidades diferenciadas en el mercado de trabajo y tendencias selectivas en él origen de clase de los estudiantes que serán atendidos por los maestros egresados de normales diferentes. 
Por otra parte, las normales se distribuyen desigualmente entre los centros urbanos de mayor tamaño (capitales departamentales) y los centros intermedios y pequeños, cuando se tiene en cuenta la calidad. Efectivamente, si se observa cómo se distribuyen las normales catalogadas como inferiores por un estudio del Ministerio de Educación y UNESCO ${ }^{28}$, se observa que en las capitales está ubicado el $10 \%$ de esas normales mientras que en el resto de los lugares está el $37 \%$. De todas maneras, sobre el $4 \%$ de normales de inferior calidad, la gran mayoría está en las ciudades intermedias y pequeñas. Además del total de normales que se hallan ubicadas en las capitales, el $36 \%$ están clasificadas como inferiores mientras que en las ciudades menores este porcentaje alcanza al $52 \%$.

Estos fenómenos revisten singular importancia para la planeación de políticas de formación de maestros, especialmente cuando se trata de reducir el número de normales como aparece conveniente en este caso, dada la disminución de la demanda y la alta proporción de normales de baja calidad. Sin embargo, la política obvia en este caso, de recortar las normales de inferior calidad, que son en su mayoría las que están ubicadas en pequeñas ciudades, traería consecuencias de gran envergadura no solamente para los procesos de movilidad social en el país, sino también para el papel que la educación debe jugar con respecto al desarrollo socio-económico nacional.

Es necesario anotar, sin embargo, un fenómeno de especial importancia que no incluye el trabajo anterior: la existencia de un gran número de escuelas rurales con primaria incompleta. Este hecho viene a calificar el exceso de oferta de maestros puesto que traslada el problema a aspectos organizacionales y de cobertura del sistema educativo. Este aspecto es analizado por Salazar Reyes en un trabajo sobre los mecanismos de ingreso y las formas de vinculación del docente en Boyacá ${ }^{29}$.

En realidad los trabajos mencionados llevan a cabo solamente un sondeo de las normales en lo que se refiere a la evolución de sus características, algunos aspectos legales y a su estratificación desde el ángulo de su calidad académica. Queda por hacer el trabajo fundamental de investigación sobre aspectos relativos a la formación de maestros y asuntos de metodología pedagógica adaptada a la variada realidad nacional, a la formación ideológica del maestro-alumno, a la organización social de la normal, y a las consecuencias sociales y educativas de su proceso de estratificación. En el mismo sentido, se hace necesario un estudio similar sobre las Facultades de educación. Por otra parte, cabría preguntarse, como tema de estudio, si tanto las normales como las Facultades de educación están realmente capacitando al maestro para capacitarse posteriormente a lo largo de su práctica profesional. Trabajos preliminares y la opinión de los mismos maestros sugieren que este no es el caso.

\section{El origen social de los maestros}

Se presentan en esta sección los hallazgos de tres estudios que tratan este tema: uno sobre el magisterio rural de un departamento ${ }^{30}$, otro sobre los maestros de seis contextos sociales de diferente grado de desarrollo ${ }^{31}$, y un tercero sobre los estudiantes de tres normales en Bogotá, Ibagué y Guasca ${ }^{32}$.

\footnotetext{
${ }^{28}$ José Alzate, Clasificación y jerarquización de las escuelas normales según criterios de calidad de los recursos humanos y de dotación, Ministerio de Educación Nacional-PNUD-UNESCO, proyecto Col. 78/003, Bogotá, 1978, Mimeo.

${ }^{29}$ Fidel Salazar Reyes, Inserción ocupacional del Educador en Boyacá, Bogotá, sin editorial, 1977.

${ }^{30}$ Gonzalo Cataño, op. cit.

${ }^{31}$ Rodrigo Parra, La profesión del maestro, op. cit

${ }^{32}$ Rodrigo Parra, Bases sociales, op. cit.

Digitalizado por RED ACADEMICA
} 
Los tres trabajos coinciden en señalar que los maestros provienen en su gran mayoría de los estratos medios y bajos de la clase media tanto rural como urbana. Los maestros hijos de obreros y de padres de origen alto son una proporción muy pequeña. Sin embargo, se presenta una inconsistencia de status en cuanto los padres de los maestros poseen una escolaridad superior al promedio de su clase. Es especialmente notoria la educación de las madres, lo que parece tener una influencia de importancia en la elección del magisterio como profesión.

Al contrario de lo que generalmente se piensa no existe una tradición familiar de la profesión: los maestros con padres maestros son una proporción mínima. Tampoco es muy elevada la proporción de maestros casados con maestras.

El estudio realizado por Gonzalo Cataño es el primero hecho en el país sobre el origen social de los maestros que ofrece información empírica y conclusiones de importancia. Muestra ya que los maestros rurales son de origen rural y sugiere el papel que las normales rurales o de pequeños pueblos tienen en la educación del área rural, así como también su juego dialéctico con el proceso de urbanización de su mentalidad inducido por la experiencia de sus estudios primarios y normalistas ${ }^{33}$. Surge también la hipótesis de que el trabajo docente implica un mayor grado de movilidad social para los maestros rurales y menor para los urbanos.

La hipótesis planteada por Cataño acerca de la existencia de movilidad social diferenciada para los maestros que se forman o que son oriundos de áreas rurales o urbanas se ve confirmada en los estudios posteriores. Efectivamente, el trabajo sobre estudiantes normalistas encuentra que la movilidad educativa es mayor para los estudiantes de normales rurales o de pequeños pueblos que para los de ciudades intermedias o que para los de Bogotá. Las explicaciones que se dan a estos fenómenos tienen que ver tanto con las condiciones sociales de los diferentes contextos que implican referentes educativos y de clase diferentes intergeneracionalmente, como a fenómenos de devaluación del valor ocupacional de la educación que es mayor en áreas urbanas que en rurales para la profesión docente. Estas hipótesis se refinan un poco más en el trabajo sobre los maestros en seis contextos con grados diferentes de desarrollo. Cuando se toma el promedio de años de educación de los maestros teniendo en cuenta el nivel de escolaridad alcanzado por el padre en los diferentes contextos sociales se puede observar que los maestros que trabajan en sitios más urbanos tienden a tener promedios superiores. Es decir, que cuando se controla la educación del padre el contexto sigue teniendo incidencia en la escolaridad de los maestros. Por otra parte, si este fenómeno se interpreta como un indicador de movilidad educativa intergeneracional se puede concluir que en términos cuantitativos es mayor la movilidad educativa de los maestros en contextos más urbanos, si bien a medida que se toman los maestros cuyos padres han alcanzado educación secundaria esa ventaja cuantitativa empieza a disminuir hasta llegar al grupo de padres con educación superior en cuyo caso se da un descenso en la escolaridad de los maestros con relación a sus progenitores. Sin embargo, estas diferencias cuantitativas pierden mucho de su peso cuando se piensa que el valor de prestigio de la educación del maestro puede ser mayor en las áreas menos urbanizadas donde la escolaridad de la población es menor y donde en términos generales el salto educativo que han dado los maestros en comparación con sus padres es de mayor significación e incluye una proporción mayor del cuerpo profesoral.

\footnotetext{
33 Esta hipótesis se halla planteada también en OFISEL, Regionalización y educación primaria en áreas rurales de Boyacá, Universidad Pedagógica y Tecnológica de Colombia, Tunja, 1976.

Digitalizado por RED ACADEMICA
} 
Puede concluirse entonces que el magisterio colombiano es originario de la clase media a diferencia de otros países en que muestran un alto componente de clase obrera $^{34}$, y que los procesos de movilidad social, específicamente educativa, son diferentes según los maestros se encuentren ejerciendo funciones profesionales en áreas de mayor o menor grado de desarrollo. Este hecho parece tener incidencia, entre otros, en el fenómeno de los maestros rurales que muestran una alta estabilidad en su historia ocupacional dentro del contexto rural, pero su peso no alcanza a causar migraciones de lo urbano hacia lo rural ni a contener una corriente migratoria de lo rural a lo urbano. Otros factores más generales de la sociedad parecen plantear fuerzas de mayor envergadura que sobrepasan los cada vez más débiles atractivos del prestigio del maestro rural en un país en proceso de urbanización. El estudio en cuestión plantea elementos de las condiciones de trabajo que son más atractivas en la ciudad, facilidades de capacitación o de asistencia a instituciones de educación superior.

Estos estudios, sin embargo, dejan sin plantear o analizan de manera muy general algunos elementos centrales de la dinámica social que sobrepasan el contexto puramente profesional de los maestros y fenómenos de tipo institucional y administrativo de la educación que sería de importancia incluir con el objetivo de maximizar la comprensión del estrecho rango de origen social de los maestros y la exclusión de otros grupos sociales.

\section{Calificación y capacitación}

No existe en realidad un estudio comprensivo y analítico de la situación educativa del magisterio nacional. Solamente análisis parciales que observan momentos específicos de forma muy general o de grupos no representativos de la población docente. Sin embargo, a través de los estudios existentes se pueden plantear algunos asuntos referentes a la calificación y capacitación del profesorado.

Desde la década de los cincuenta la situación escolar del magisterio ha mejorado notablemente. En 1953 el 65\% de los maestros no tenían ningún grado y actualmente esa cifra debe estar alrededor del $20 \%$ o menos. La diferencia para los maestros urbanos y rurales favorece siempre a los urbanos ${ }^{35}$ que cuentan con personal más calificado y mejor escalafonado. Existen diferencias marcadas en la forma como se agrupan por salarios los maestros en las regiones del país, agrupación que ha ido variando entre regiones con el tiempo siguiendo en general las líneas del desarrollo regional ${ }^{36}$.

En 1968, "El nivel educativo de los maestros rurales de Boyacá es bastante desigual: $2.9 \%$ no pasó de la primaria; uno poco más de una cuarta parte (27\%) no terminó la enseñanza secundaria, completándola sólo un $5.1 \%$; y el $64 \%$ estuvo en la normal, ocupando los normalistas rurales un lugar reducido. Al comparar estos datos con los del área urbana se encuentra que ésta posee un magisterio con una mayor preparación, siendo importante anotar el mayor porcentaje de normalistas rurales que parece reflejar una huída de estos profesionales del campo hacia las ciudades".

\footnotetext{
${ }^{34}$ Ida Bergerm, Les instituteurs done génération a laatre, Paris, Presses Universitaires de France, 1979. Orlando Albornoz, El maestro y la educación en La sociedad Venezolana, Caracas. 1965. sin editorial. En cambio el caso brasileño presenta características similares a las de Colombia, cfr. Luis Pereira, O magisterio Primario numa sociedade de classes. Sao Paulo, Livraria Pioneira Editora, 1969.

${ }^{35}$ Gonzalo Cataño, op. cit.

${ }^{36}$ Gonzalo Cataño, op. cit., Misión Economía y Humanismo, op. cit., Rodrigo Parra, La educación rural en la zona cafetera colombiana, Buenos Aires, UNESCO-CEPAL-PNUD, proyecto "Desarrollo y Educación en América Latina y el Caribe". 1978.
} 
En 1979 la situación ha mejorado sustancialmente, aunque los datos que se citan en seguida son dados por los maestros mismos y es necesario tomarlos con cautela ${ }^{37}$.

La totalidad de los maestros afirma haber terminado la escuela primaria. La gran mayoría de los encuestados dicen haber cursado la educación secundaria y haberse graduado en este nivel aunque la proporción es mayor entre los maestros que trabajan en contextos urbanos que en rurales. La escolaridad superior desciende a medida que el contexto de trabajo de los docentes se hace más rural.

En términos generales el tipo de educación secundaria que tienen los maestros y su entrenamiento pedagógico parece estar muy condicionado por las oportunidades educativas que existen en su contexto de estudio. Este fenómeno se relaciona con la distribución espacial de las normales y Facultades de educación y debe tenerse en cuenta cuando se planifica la fundación o discontinuación de establecimientos pedagógicos. Sin embargo, debe también tenerse en cuenta que las migraciones estudiantiles de los maestros afectan este fenómeno y pueden llegar a incidir de manera significativa.

En general los maestros piensan que los estudios de las normales no capacitan para enseñar sin la ayuda de la capacitación. La clara conciencia que los maestros expresan de este hecho hace aún más importante conocer, aunque sea de manera general, las formas que utilizan para ponerse al día con los avances del conocimiento en su área profesional.

Aproximadamente la mitad de los maestros afirma que se actualizan por medio de lecturas. Una segunda forma de actualización son los cursos de capacitación y una tercera modalidad está constituida por el trabajo en grupo.

En términos globales las opiniones de los maestros configuran un cuadro que se centra en la desconexión entre el entrenamiento del docente y su realidad laboral en dos sentidos: a) entre la imagen de participante en los procesos comunitarios y su formación fundamentalmente dirigida al trabajo intraescolar y b) entre la capacitación y las necesidades prácticas encontradas en los contextos sociales específicos, especialmente en el caso de los contextos menos urbanos. Estas ideas son, por otra parte, expresadas de manera vaga y general, pero su percepción es de todas maneras anotada consistentemente.

\section{Migraciones profesionales}

Poco se sabe sobre los movimientos migratorios de los maestros en cumplimiento de su actividad profesional. Este es un tema de especial importancia no solamente desde el punto de vista de la planeación educativa y de la política de capacitación magisterial sino también desde el ángulo del rendimiento escolar y la movilidad social de los docentes, además del interés que despierta el tema dentro de la sociología de las profesiones.

Se presentan en seguida algunos hallazgos de un estudio que es todavía muy general y basado en una muestra que no debe considerarse representativa de los maestros colombianos pero puede ser de utilidad para abrir la discusión sobre el tema ${ }^{38}$.

\footnotetext{
${ }^{37}$ Rodrigo Parra, La profesión del maestro, op. cit.

${ }^{38}$ Rodrigo Parra, La profesión del Maestro. op. cit.

Digitalizado por RED ACADEMICA
} 
El trabajo, basándose en una historia ocupacional de maestros de seis contextos sociales con diferente grado de desarrollo económico ${ }^{39}$, planteados tipos de migración: la migración con propósitos educativos y la migración con propósitos laborales.

Con respecto a las migraciones con propósitos educativos, la tendencia general que aparece más clara a primera vista es hacia la estabilidad, o sea que los maestros tienden a estudiar y trabajar dentro del mismo contexto. Sin embargo, por debajo de esta estabilidad fluyen diversas corrientes dignas de mencionarse porque conforman patrones de conducta. El primero de esos patrones está constituido por una corriente migratoria de estudiantes de primaria de contextos rurales hacia urbanos, al pasar a escuelas de nivel secundario. El fenómeno es correlativo e inverso con relación a los contextos rurales. El segundo patrón de conducta sugiere que aunque se de una tendencia a estudiar la secundaria en contextos más urbanos y a pesar de que una proporción de los migrantes permanezcan después trabajando en con-textos urbanos, una alta proporción de los que migran a estudiar regresan a enseñar en contextos rurales.

En términos generales tres de cada cuatro maestros han permanecido durante toda su historia ocupacional dentro del mismo contexto. Solamente el $15 \%$ de los educadores han realizado más de un movimiento entre con-textos. Estos datos sugieren que la estabilidad en el contexto de trabajo es mayor de lo que generalmente se piensa, si bien la migración entre contextos no es igual a la migración de un sitio a otro que puede darse con mayor o menor fuerza dentro del mismo contexto.

En todos los contextos la mayoría de los maestros se han mantenido trabajando dentro del mismo contexto social. El porcentaje de estabilidad es, sin embargo, mayor en los contextos rurales y creciente en el orden descendente de desarrollo relativo. Los contextos urbanos reciben flujos de importancia de docentes que empezaron su actividad profesional en contextos rurales: aproximadamente uno de cada cuatro maestros en el industrial no marginal y uno de cada tres en el marginal y en el no industrial. El urbano no industrial contribuye también al magisterio del contexto industrial no marginal.

Adicionalmente, es interesante observar los movimientos migratorios que se dan al interior de los contextos agrícolas entre la cabecera municipal y las zonas más estrictamente rurales. El primer hecho relevante es la existencia de un fuerte intercambio de migraciones en ambos sentidos: en las cabeceras, de cada diez maestros seis empezaron y están ahora trabajando allí mientras cuatro provienen del área rural. En el área rural el fenómeno es el mismo: de cada diez maestros seis empezaron y están ahora en la zona mientras los cuatro restantes provienen de las cabeceras municipales. En las zonas rurales disminuye sensiblemente la proporción de maestros que empezaron y continúan trabajando allí a medida que se baja en el gradiente de desarrollo.

En resumen, puede concluirse que la tendencia fundamental de los maestros es la estabilidad en lo que se refiere a las fronteras intercontextuales. Las líneas principales de migración intercontextual se presentan de los con-textos agrícolas hacia los urbanos y del contexto urbano no industrial hacia los urbanos industriales y hacia los agrícolas. Existe un abundante y frecuente flujo intracontextual en los contextos agrícolas entre cabeceras municipales y área propiamente rural.

Es de interés anotar que tanto este trabajo como el de Gonzalo Cataño tienden g comprobar las hipótesis sobre el origen predominantemente rural de los maestros rurales

\footnotetext{
${ }^{39}$ Urbano industrial marginal y no marginal, urbano no industrial, agrícola industrial, agrícola empresarial y campesino.
} 
y sobre su urbanización educativa. Por otra parte, en un trabajo en proceso, Himelda Martínez muestra la importancia de las corrientes migratorias dentro de lo rural y el papel que elementos del desarrollo como la red vial juegan en la movilidad física y social de los maestros ${ }^{40}$.

\section{EI maestro y el rendimiento escolar}

Este tema ha sido tratado de manera suscinta en un trabajo de Harold Banguero dedicado centralmente al análisis de las relaciones entre nutrición y escolaridad ${ }^{41}$, con base en una muestra de escuelas en cinco ciudades.

El estudio presenta un análisis de covarianza para elucidar los determinantes de la calidad del profesor $^{42}$ y concluye que la mayor edad del profesor presenta un impacto negativo sobre su calidad, la experiencia laboral un efecto positivo y que el sexo no influye, que la calidad de los profesores del sector privado es significativamente inferior que la del sector público y que la inestabilidad en la escuela influye negativamente. La experiencia y la estabilidad se constituyen en los factores de mayor importancia.

A su vez, el estudio estima por medio de regresiones simples el impacto de una serie de variables en el rendimiento escolar de los alumnos ${ }^{43}$. Entre las variables que se refieren al maestro se encuentra que la edad del profesor influye en el sentido de que los alumnos con profesores más jóvenes rinden más, los alumnos con profesores especializados en pedagogía rinden más, pero la estabilidad del maestro no parece tener efecto en el rendimiento del escolar.

El estudio tiene el valor de ser un intento aislado en el país de enfocar cuantitativamente las relaciones entre la calidad del profesor y el rendimiento escolar del alumno. Sus conclusiones, sin embargo, son muy generales y tal vez el hallazgo de mayor interés se refiera a la utilidad de la formación pedagógica del maestro. Es por lo menos discutible tomar el status socio-económico del profesor, medido en términos de sus posesiones materiales, como una medida de su calidad docente. Es desconcertante que la estabilidad del profesor en la escuela califique su calidad pero no tenga efecto en el rendimiento de los estudiantes y que la edad del profesor esté inversamente relacionada con su calidad al tiempo que la mayor experiencia determine un mayor rendimiento escolar. A su vez, el estudio excluye, debido a su diseño, elementos muy importantes del proceso de enseñanza-aprendizaje, como los aspectos no cognoscitivos, las formas que el maestro utiliza para enseñar y se centra en los contenidos de la enseñanza ${ }^{44}$. La forma empleada de medir el rendimiento escolar genera, por otra parte, un vacío entre los planteamientos empleados actualmente en la capacitación de los maestros que se centran en la conducta en el aula, en la manera de enseñar y aprender, y la forma de evaluar el aprendizaje que se orienta a medir el banco de información que tienen los alumnos y, en el mejor de los casos, los procesos de raciocinio, pero que excluye patrones de conducta, por ejemplo, hacia el autoritarismo o la participación. Adicionalmente, este tipo de análisis excluye el estudio de otras funciones del maestro que tienen que ver con la calidad de su acción, como sus relaciones con la comunidad, su

\footnotetext{
${ }^{40}$ Himelda Martínez Z., El Maestro Rural Colombiano, en elaboración.

${ }^{41} 24$ H. Banguero. et al., Nutrición y escolaridad: el caso colombiano, CEDE-ECIEL, Bogotá, 1979, Documento CEDE No. 059.

${ }^{42}$ La calidad del profesor es medida por medio de las siguientes variables: índice de status socio-económico, nivel de estudios y horas semanales en la escuela.

${ }^{43}$ El rendimiento es medido por medio de pruebas en lectura y ciencias naturales.

${ }^{44}$ Ver por ejemplo Comentarios al respecto en Beatrice Avalos y Wodi Hoddod, A Review of Teacher Effectiveness Research in Africa, India, Latin America, Middle Est, Malaysia, Philiippines and Thailand: Synthesis of Results, Otawa, IDRC, 1979. Abraham Magendzo y Ricardo Haria, Research mio Teacher Effectiveness in Latin America, Santiago, PIIE, 1978, Mimeo.
} 
papel de vínculo entre el Estado y la localidad especialmente en áreas menos urbanizadas y en participación en actividades sociales, económicas de las gentes, así como también sus contactos con los padres de familia. En descargo parece oportuno decir que este tipo de fenómenos puede ser más eficientemente estudiado con otras técnicas de investigación social.

\section{La ruptura entre la realidad social, la imagen y la práctica}

Uno de los elementos básicos constitutivos de una profesión es la conformación de una imagen que le confiera sentido social, que la dignifique y le de un código de conducta dentro del cual debe desarrollar su actividad específica. La importancia de esta imagen es más sentida en ciertas profesiones que por su naturaleza juegan papeles claves en la división social del trabajo y que han ido creando una noción de vocación y de servicio a la sociedad, como el sacerdocio, la medicina y el magisterio. La naturaleza de esta imagen, sus cambios y su vinculación con los procesos de cambio de la sociedad guardan una estrecha relación con la efectividad de sus profesionales ${ }^{45}$. Se presentan en seguida las ideas centrales de varios trabajos que tratan algunos aspectos de este tema ${ }^{46}$.

Los trabajos tratan dos temas centrales: 1) la ruptura que se presenta entre la imagen sagrada que los maestros tienen de su profesión, la realidad social cambiante a través del proceso de división del trabajo y la práctica de la docencia; 2) el segundo tema se refiere al conflicto que viven los maestros entre dos funciones fundamentales del quehacer docente, la función social formadora de ciudadanos idóneos dentro del marco ideológico predominante de la sociedad y la función pedagógica que enfatiza la maximización del aprendizaje y que en sus versiones modernas implica el desarrollo de la creatividad y el desarrollo de actitudes críticas hacia el conocimiento.

1. La concepción predominante entre los maestros es la que corresponde a una vocación de servicio a la sociedad, a una misión que da sentido a la vida y que debe expresarse concretamente en su vinculación con la comunidad. Esta imagen choca actualmente con situaciones de desarrollo social generadas por el proceso de urbanización e industrialización que la hace ineficaz y produce reacciones anónimas en el maestro. Muchas de sus funciones de liderazgo son ahora llenadas por otras instituciones o servicios que se han especializado y que entran a "recortar" las posibilidades de liderazgo del maestro. Algunas comunidades se organizan por sí mismas o a través de organismos estatales o privados y restringen el ámbito de trabajo tradicional del docente, otras rechazan su acción extraescolar y a veces docente por su falta de relación con la vida práctica de la comunidad o por procesos especiales de integración (comunidades marginadas urbanas, por ejemplo) ${ }^{47}$.

Dieter $\mathrm{Paas}^{48}$ en un trabajo de mucho interés sobre los maestros rurales sostiene algunos de estos puntos de vista desde la opinión de los maestros y sus obstáculos en el trabajo con las comunidades y, de alguna manera, desde las actitudes de miembros de las comunidades hacia el trabajo del docente. Aunque el trabajo de Paas incluye la

\footnotetext{
${ }^{45}$ Ver por ejemplo un análisis del papel jugado por la imagen profesional de los maestros en Venezuela en Orlando Albornoz, $E l$ Maestro y la Educación en la Sociedad Venezolana, Caracas, 1965, sin editorial.

${ }^{46}$ Milcíades Chávez, et al., "Educación y estructura social en Nariño"; Ernesto Guhl, et al., "La educación rural en Caldas"; CIDA, "Educación y Tenencia de la tierra"; Virginia Gutiérrez de Pineda, "Educación y mundo rural"; William C. Sayres, "La educación en una comunidad rural colombiana: el caso de Zarzal, Cauca"; Orlando Fals Borda, "La educación formal en Saucío"; Gerardo y Alicia Reichel-Dolmatoff, "La enseñanza formal en Aritama". Todos estos trabajos en Gonzalo Cataño, ed., Educación y Sociedad en Colombia, Bogotá, Universidad Pedagógica Nacional, 1973 y otros ya citados.

${ }^{47}$ Por ejemplo el trabajo en proceso de Juan Carlos Tedesco, Rodrigo Parra y Maria Elvira Carvajal, La escuela en un barrio marginal de Bogotá.

${ }^{48}$ Dieter Paas, op. cit.

Digitalizado por RED ACADEMICA
} 
presentación de varias formas de vinculación del maestro con la comunidad parece de utilidad hacer resaltar un aspecto específico con una cita de la opinión de un maestro:

"Es otra dificultad del maestro que no está capacitado en técnicas agropecuarias... En agropecuarias, ¿qué le enseñan a uno en la normal? Ahí le enseñan a analizar el suelo, por ejemplo, pero teóricamente, no lo hacen prácticamente. (...) Se necesita capacitación pero de algo, práctico. Porque los padres de familia se acomodan más con un maestro que no está continuamente dando clases en su aula, que vaya a toda la vereda colaborando con los padres de familia".

Esa cita es de mucho interés porque plantea dos conflictos centrales a la actividad magisterial, su conflicto entre la función central de enseñanza en el aula y su función de líder comunitario, por una parte, y su conflicto entre el tipo de enseñanza que recibe en la normal y el que le exige la relación con comunidades rurales, por otra.

Pero, adicionalmente, su ubicación en la comunidad no obedece solamente a su conocimiento académico, sino también a su ubicación social y al entendimiento' de los papeles que puede jugar dentro de la dinámica social, como lo muestra la siguiente afirmación del mismo autor:

"La función de liderazgo del maestro se origina entonces, no tanto en su mayor elocuencia o fuerza de convicción, sino es más bien su posición favorable dentro de la red de relaciones públicas de favorecedores y clientela, su mayor cercanía de los políticos de partido con poder de decisión sobre recursos públicos, lo que mueve a la comunidad rural a escogerlo como su delegado".

Otro tipo de problema relacionado con la práctica profesional es el planteado entre el currículo, su aplicación y la realidad social. Myriam Ferro ${ }^{49}$, en un vívido recuento de la vida de una maestra rural lo plantea así, en boca de la maestra:

"Los objetivos de la educación rural son: que el niño conozca el medio en que vive, se preocupe en estudiar y trate de adelantarse, terminando con el conformismo en que viven. Pero este objetivo no se cumple porque la educación es igual en contenido para todos, y uno tiene poco tiempo para pensar en otra cosa que no sea cumplir con los programas; además son varios cursos simultáneos que tenemos que atender. Los programas que tenemos que cumplir en las diversas materias son tan antiguos, tan rígidos, tan largos, que a veces me da la impresión de que quien lo ha hecho no conoce cuál es la situación que vive el niño en el campo, no va de acuerdo con sus intereses y es impositivo, pues toca cumplirlo, lo que hace para nosotras más difícil lograr el aprendizaje".

Por supuesto, en las relaciones entre el maestro y la comunidad se presenta una extensa gama de situaciones, desde el ejercicio del liderazgo por parte del maestro hasta su desinterés más extremo, pasando por actitudes racistas, supervaloración del trabajo intelectual o escolar y subvaloración del conocimiento práctico, rechazo de parte de la comunidad o inserción del maestro en ella, como puede verse en los estudios de comunidad mencionados en la cita.

El interés científico para la comprensión del papel del maestro, su relación con la imagen profesional, los cambios del contexto social y su práctica docente, se

\footnotetext{
${ }^{49}$ Myriam Ferro, op. cit.
} 
incrementaría en los estudios etnográficos si superarán el nivel puramente descriptivo y entraran en una etapa de análisis explicativo de las razones por las cuales se dan esos fenómenos, sus cambios con el proceso de división del trabajo, sin abandonar, por supuesto, su énfasis antropológico.

2. En lo que respecta al conflicto que les plantea a los maestros su relación con las dos funciones, la social y la pedagógica, la mayoría de los maestros responden afirmativamente a ambas tendencias aunque en mayor proporción hacia la función pedagógica (83\%) que hacia la social (70\%). Esto implica que el $70 \%$ de los profesores al afirmar ambos objetivos están expresando una imagen de su profesión que contiene elementos centrales que se oponen entre sí. El estudio de Parra plantea tres variables que condicionan la presencia de este fenómeno: los profesores más jóvenes tienden a escoger en mayor proporción la función pedagógica que los de mayor edad. Los maestros que trabajan en contextos sociales más urbanizados tienden también a decidirse por una imagen profesional más inclinada hacia la función pedagógica. A su vez, los datos indican que cuando el profesor percibe que el origen social de 'sus alumnos es de clase media alta o tiende a dar más importancia al objetivo pedagógico que cuando los percibe como de clase baja.

El breve análisis de este tema termina afirmando que los conflictos que surgen en la imagen que el maestro tiene de su actividad profesional contribuyen a confundirlo más y que cuando se piensa en la planeación de reformas curriculares o en el diseño de estrategias de capacitación deben considerarse estos fenómenos y hacer un esfuerzo por esclarecer los objetivos en términos de la situación global del docente con el propósito de reducir las disonancias creadas por demandas encontradas.

El estudio de la imagen del maestro, como uno de los componentes de la ideología docente, apenas ha sido realizado en sus planteos iniciales. Este es un tema que demanda una mayor profundización y la inclusión de otros aspectos, específicamente el de la relación de la imagen con la estructura política, el de las teorías de la relación entre maestro y alumno y el de la vinculación con la comunidad. Es un campo apenas inicialmente desbrozado que merece mayor atención.

\section{Los agentes educativos en la educación no formal}

Es muy escasa la investigación sobre maestros en situaciones de educación no formal, a pesar de que esta modalidad docente ha venido tomando un auge de importancia en las últimas décadas, especialmente a partir de los años cincuenta. Existe, sin embargo, un estudio pionero que marca algunas líneas de interés en este campo realizado por CEDEN y publicado en $1975^{50}$. Este trabajo centra su interés en tres aspectos de la actividad docente: 1) características de los maestros, 2) capacitación de los agentes educativos y, 3) técnicas de enseñanza más empleadas.

El estudio encontró que, al contrario de la educación formal, el grupo mayoritario de los agentes educativos no formales está conformado por voluntarios no remunerados y que la proporción de remunerados aumenta a medida que es mayor el desarrollo de las zonas en que trabajan. Los voluntarios, en cambio, parecen concentrarse en mayor medida en las zonas más urbanizadas. Aunque los usuarios (estudiantes) de la educación no formal pertenecen a grupos sociales con ingresos inferiores a los que asisten a la escuela formal y muestran características educativas muy inferiores, los mecanismos de concentración

\footnotetext{
${ }^{50}$ Wilson Velandia, op. cit.

Digitalizado por RED ACADEMICA
} 
educativa a través del desarrollo desigual y la urbanización siguen operando en la educación no formal de la misma manera que en la formal.

Las características educativas de los maestros en el sistema no formal son, en términos generales, comparables a los del sistema formal. Sin embargo, se notan algunas diferencias que son marcadas por el hecho de que la proporción de maestros en el sistema no formal con educación primaria y secundaria es inferior al del formal pero superior en el nivel universitario. Este último hecho, aduce el informe, puede deberse a la naturaleza de las actividades no formales más conectadas con el trabajo, y a dificultades de empleo por parte de egresados universitarios. Por otra parte, los voluntarios tienen generalmente dedicaciones parciales mientras que los remunerados trabajan de tiempo completo.

Los sistemas de capacitación son aún muy incipientes en la educación no formal y aunque existe una buena proporción de casos de capacitación, en general no hay programas que dirijan la capacitación. Los medios más empleados para capacitar difieren entre los voluntarios (entrenamiento en el puesto de trabajo) y los remunerados (cursillos, seminarios, talleres). La utilización de entrenamiento formal para la vinculación y el material escrito son poco empleados como sistemas de capacitación.

En general las metodologías de enseñanza pueden considerarse novedosas o innovativas en un $50 \%$ (individualizada, juegos de aprendizaje, entre compañeros, dramatizaciones, prácticas y demostraciones), mientras que el resto se pueden clasificar como tradicionales (conferencia, clase magistral y otras). Las metodologías tradicionales tienden a predominar en las grandes ciudades.

Con respecto a las formas que toma la relación maestro-alumno se encuentra que sobresale la situación en que el docente asume el papel predominante y el usuario juega un papel individual y en una segunda instancia se ubica la situación en que el maestro juega el papel de recurso y el usuario trabaja en grupo o individualmente.

El estudio de CEDEN ha cumplido una función de importancia como iniciador de la investigación sobre un tipo especial de maestros que aunque ha tenido un antecedente en el área de la extensión agrícola y otras áreas ${ }^{51}$, no se había realizado desde el ángulo de un estudio social más comprensivo. Desafortunadamente no ha suscitado estudios posteriores de envergadura que continúen profundizando los hallazgos iniciales. El trabajo, debido posiblemente a su naturaleza exploratoria, se definió como una indagación descriptiva de los proyectos de educación no formal. Sin embargo, subyace un planteamiento que tiene sus raíces en teorías del desarrollo desigual y de la división social del trabajo. Esos planteamientos, sin embargo, no se emplean para lograr una explicación de los fenómenos que se describen sino fundamentalmente para ofrecer una taxonomía social que enriqueciera la descripción. Queda para estudios posteriores plantearse una pregunta fundamental que surge de la lectura: ¿qué razones de tipo social, económico o político llevan a que un tipo de educación diseñado explícitamente para llegar a grupos sociales y áreas espaciales donde no penetra la educación formal, siga los mismos lineamientos de concentración y, en algunos casos, discriminación grupal en la escuela? ¿Esas razones se encuentran en las formas como han sido diseñados y organizados los programas de educación no formal, o acaso en las características y circunstancias laborales de los maestros de este tipo de educación?

\footnotetext{
${ }^{51}$ Ver por ejemplo, Informe del Banco Mundial, Desarrollo económico de Colombia: problemas y perspectivas, Bogotá. Banco Popular. 1970.
} 


\section{Conclusiones}

Los conocimientos que se tienen actualmente, basados en estudios sociales, sobre el maestro colombiano son en general fragmentarios. Aunque algunos de los estudios revisados intentan una aproximación global a la situación del maestro, la mayoría de ellos trabajan solamente puntos específicos no siempre vinculados con los procesos generales de la sociedad. Hace falta una teoría más comprensiva del maestro colombiano. En buena parte esto se debe también a que la información existente sobre maestros es fragmentaria. Por otra parte, debido a la naturaleza exploratoria de los estudios, muy pocos alcanzan un grado de profundidad tal que esclarezcan a satisfacción los asuntos centrales que plantean los temas aislados o específicos que son su objeto. Esto llevaría a la necesidad de priorizar cuatro tipos de estudios:

1. Trabajos de tipo teórico y sociohistórico capaces de seleccionar y presentar de manera orgánica los fenómenos centrales de la relación maestro-sociedad que sirvan como base o que den lugar a una comprensión amplia de estos problemas.

2. Trabajos que puedan recopilar con un grado aceptable de validez información cuantitativa general sobre los maestros, capaces de proveer una base para generalizar empíricamente sobre algunos procesos centrales de la situación del docente nacional y dar pie al establecimiento de muestras estadísticamente aceptables.

3. Estudios de observación cualitativa en profundidad en algunas áreas de la educación en que interviene el maestro como personaje central y que son prácticamente desconocidas. Un ejemplo de este tipo de estudios podría ser dado por la observación con técnicas antropológicas de la escuela. Es muy poco lo que se sabe, desde el punto de vista de los estudios sociales, sobre cómo funciona la escuela, cuáles son sus formas de organización social, métodos pedagógicos, cumplimiento de funciones declaradas de aprendizaje, como la alfabetización en uno o dos años de los alumnos, transmisión de valores sociales, cambios y efectos del currículo en las diferentes regiones del país y, por supuesto, el papel que los maestros juegan en este contexto.

4. Un cuarto tipo de estudios que convendría priorizar es el de trabajos monográficos sobre aspectos poco investigados hasta ahora. Ellos contribuirian a llenar vacíos de información que impiden una comprensión más clara y precisa sobre los maestros colombianos. Se enuncian a continuación algunos de los tópicos de mayor importancia.

Existe un gran vacío en el estudio de la organización gremial de los maestros. Este vacío es especialmente notorio puesto que FECODE, el organismo sindical de los maestros, ha jugado un papel de gran importancia en el país. Es de especial relevancia estudiar y comprender mejor la forma y los contenidos sustanciales que los docentes como un cuerpo plantean en sus relaciones con el Estado. Una forma posible de aproximación se podría dar a través del análisis de los diferentes proyectos de estatuto del profesorado que se han tramitado con diversos resultados en las últimas décadas. Sería de mucho interés conocer las reivindicaciones sociales planteadas por los maestros en estos estatutos distinguiendo entre la vertiente que se refiere a las condiciones de trabajo de sus miembros y la que hace referencia a mejoras educativas y al papel de la educación y del educador en la sociedad. De un análisis de este tipo saldrían elementos centrales de la estructura ideológica del movimiento magisterial. Por otra parte, hace falta 
un estudio serio sobre las condiciones sociales que permitieron el surgimiento del sindicalismo docente, su auge y su crisis, así como también de las líneas centrales de su organización interna como movimiento que en su momento de mayor importancia pareció desbordar los planteamientos puramente reivindicativos y tomar ribetes de movimiento social de carácter más amplio.

Otra área de importancia que no ha sido cubierta por los estudios sobre el docente colombiano es la que se refiere a las relaciones entre la escuela, el maestro y la generación de valores sociales en los alumnos. Esta franja de investigación se dirige al esclarecimiento de la visión del mundo, de las formas fundamentales de relación dentro de la sociedad, la imagen de nación, Estado, grupos sociales, la naturaleza autoritaria o democrática de confrontar las interacciones sociales que el maestro y las formas de organización social de la escuela están trasmitiendo al alumno. Esta zona problemática puede, por supuesto, desde el punto de vista teórico, enmarcarse dentro del análisis de la estructura ideológica. Habría que distinguir, sin embargo, dos aspectos centrales pero diferentes: el análisis de las expresiones o imágenes verbales que exteriorizan tanto maestros como alumnos y las formas de conducta que transmiten los educadores a través de la práctica docente y de la organización escolar.

Del punto anterior se desprende la importancia de estudiar otro aspecto de la relación maestro-alumno: los métodos pedagógicos que efectivamente emplea el maestro en contraste con las expresiones verbales o teóricas sobre didáctica. Aquí resulta de interés observar ambas conductas para evaluar el efecto que realmente producen en el aula las enseñanzas teóricas de la pedagogía en las escuelas normales $u$ otras formas de entrenamiento magisterial y su consistencia en la práctica. Es muy posible que su inconsistencia sea muy fuerte y que la transmisión de formas de conducta se deba más a lo que el maestro hace que a lo que dice. Fenómenos de especial relevancia pueden estar en juego en este proceso, como la conformación de valores básicos para el funcionamiento de una sociedad democrática, participativa o tendencias hacia una imagen autoritaria de la vida social. Este tipo de estudios requiere, por supuesto, de técnicas de investigación basadas centralmente en la observación del desarrollo de la vida escolar donde un intento de enfoque con herramientas de observación demasiado estructuradas de antemano podría correr el riesgo de dejar por fuera los fenómenos de mayor relevancia. A nivel histórico de las ideas pedagógicas y de algunos de sus efectos en la práctica docente es necesario mencionar los trabajos de Olga Lucía Zuluaga sobre el siglo $\mathrm{XIX}^{52}$. Estos trabajos resultan de especial interés por cuanto dibujan un planteamiento teórico, analizan por medio del contenido de los textos la influencia de diversas orientaciones didácticas en la escuela, y diseñan una forma de ver las ideas pedagógicas en el país. Este ejercicio debe necesariamente anteceder a la observación de la práctica docente de los maestros y sus consecuencias en la generación de conductas y valores en los alumnos en la escuela colombiana contemporánea.

Es necesario esclarecer el efecto que en su actividad específica de docente tiene la adjudicación de una multiplicidad de funciones al maestro. Cómo reparte el maestro su tiempo y su eficiencia entre sus funciones de enseñanza, de líder comunitario, de representante del Estado especialmente en las áreas rurales, de consejero escolar con los padres de familia. La importancia que se le puede dar a cada una de estas funciones varía con las formas y grados de desarrollo de las regiones en que el maestro se desempeña, lo que lleva a pensar en la importancia de estudiar el fenómeno teniendo en

\footnotetext{
${ }^{52}$ Olga Lucía Zuluaga. Didáctica y conocimiento, Medellín, Universidad de Antioquia. CIED, 1977. Colombia: Dos modelos de Práctica Pedagógica durante el siglo XIX. Medellín. Universidad de Antioquia, CIED. 1978.
} 
cuenta el proceso de desarrollo desigual del país. Estudios de este tipo podrían tener una inmensa repercusión en la planeación y la política educativa, en reformas curriculares, en los sistemas de evaluación y en la formación y capacitación de los maestros, o en la acumulación de conocimientos que conduzcan a la formulación de bases sociales para un proceso de descentralización de la educación.

Sin dementar la utilidad y la conveniencia de estudios sofisticados metodológicamente, dirigidos a esclarecer los condicionantes del rendimiento escolar, parece conveniente meditar en problemas relacionados con este tópico que pueden tener tanta o mayor prioridad en Colombia. En un país en el que una proporción alta de la población es todavía analfabeta parece ineludible aproximarse a la comprensión y análisis de la posible participación que a la escuela y al maestro le cabe en este fenómeno. Una de las funciones de la escuela primaria es la de alfabetizar a los alumnos. Pero cuando para una buena parte de la población escolar el paso por esta institución educativa se reduce a uno o dos años, esta función adquiere un carácter de la máxima importancia. En estas circunstancias el estudio de los condicionantes de la alfabetización en la escuela, los factores pedagógicos, sociales y de otra índole, debe convertirse en un área prioritaria de investigación. Es necesario saber cuál es el peso de estos condicionantes, los elementos pedagógicos que debe manejar el maestro, los condicionantes sociales que producen el analfabetismo por desuso 'y el conjunto de las causas por las cuales la escuela no alfabetiza a una porción de sus alumnos en uno o dos años, como muestra un estudio realizado en una escuela de Bogotá $^{53}$.

Otros tipos de investigaciones que aparecen como prioritarias serían las siguientes: un estudio en profundidad sobre las normales y las facultades de educación que analizara las formas de enseñanza, técnicas pedagógicas, teoría en yoga y su aplicación por parte de los maestros y de los alumnos, organización social de normales y facultades de educación, imagen del maestro que se trasmite y sus consecuencias en la forma de llevar a cabo la docencia y las relaciones con la comunidad, el currículo y su relación con las formas de desarrollo del país. Desde otro ángulo, es necesario saber cuál es, en diferentes contextos sociales, la imagen que las comunidades tienen sobre las funciones del maestro, puesto que parece válido plantear la hipótesis de que la imagen tradicional del maestro rural ha sufrido una serie de modificaciones inducidas por el proceso de división del trabajo. Este tipo de conocimiento puede ser muy valioso para la formación de los maestros ya que puede incrementar su eficiencia en la labor docente y disminuir conflictos derivados de su auto imagen y la confrontación con la realidad. En este punto es de especial importancia el estudio de las denominadas materias vínculo como vehículo de comunicación entre la formación de los docentes y su enraizamiento en la realidad del país. Estos estudios pueden mirarse también como elementos para una política de capacitación, conjuntamente con estudios que analicen problemas específicos de esta forma de entrenamiento de los maestros.

Dos fenómenos adicionales que ameritan estudio están definidos, uno por la situación económica de los maestros, sus ingresos, trabajos adicionales, y la forma en que su ubicación salarial afecta su actividad, su rendimiento, su estabilidad dentro de la carrera docente y sus actitudes profesionales. El otro estudio tiene que ven con las diferencias que marcan el trabajo en distintos niveles y tipos de escolaridad. Las investigaciones existentes se refieren principalmente al nivel primario y sería de importancia conocer los

\footnotetext{
${ }^{53}$ María Elvira Carvajal, Alfabetización infantil en barrios marginales de Bogotá, UNESCO- CEPAL-PNUD, trabajo presentado al tercer seminario regional del proyecto "Desarrollo y Educación en América Latina y el Caribe" sobre "Condiciones sociales del analfabetismo y el cumplimiento de la obligatoriedad escolar", Quito. 1979.

Digitalizado por RED ACADEMICA
} 
problemas específicos y la naturaleza de los docentes en otros niveles, como el secundario, preescolar, maestro con técnicas a distancia, educación técnica y universitaria.

En resumen, la consideración de lo que se ha hecho en términos de estudios sociales sobre el magisterio nacional deja la impresión de que la investigación de esta área de la realidad nacional apenas empieza. Es notoria la ausencia fundamental de un estudio general, amplio y comprensivo, que unifique teóricamente los diferentes aspectos que afectan la actividad del maestro así como los cambios sociales que induce el docente colombiano. El mismo podría constituir un punto de partida que sirviera para profundizar en temas específicos sin que éstos perdieran de vista la visión global dentro de la cual se enmarcan. 